\title{
Antioxidative and Anti-Inflammatory Effects of Lactobacillus plantarum ZS62 on Alcohol-Induced Subacute Hepatic Damage
}

\author{
Yi Gan, ${ }^{1}$ Xiufeng Chen, ${ }^{2}$ Ruokun Yi, ${ }^{1}$ and Xin Zhao $\mathbb{D}^{1}$ \\ ${ }^{1}$ Chongqing Collaborative Innovation Center for Functional Food, Chongqing Engineering Research Center of Functional Food, \\ Chongqing Engineering Laboratory for Research and Development of Functional Food, Chongqing University of Education, \\ Chongqing 400067, China \\ ${ }^{2}$ Gastrointestinal Cancer Center, Chongqing University Cancer Hospital, Chongqing 400030, China
}

Correspondence should be addressed to Xin Zhao; zhaoxin@cque.edu.cn

Received 29 September 2021; Accepted 24 November 2021; Published 6 December 2021

Academic Editor: Víctor M. Mendoza-Núñez

Copyright ( 2021 Yi Gan et al. This is an open access article distributed under the Creative Commons Attribution License, which permits unrestricted use, distribution, and reproduction in any medium, provided the original work is properly cited.

Lactobacillus plantarum ZS62 is a newly isolated strain from naturally fermented yogurt that might offer some beneficial effects in the setting of alcohol-induced subacute liver injury. The liver-protective effect of $L$. plantarum ZS62 was investigated by gavage feeding of mice with this Lactobacillus strain $\left(1 \times 10^{9} \mathrm{CFU} / \mathrm{kg}_{\mathrm{BW}}\right)$ before alcohol administration daily for 7 days. We then compared hepatic morphology, liver function indexes, liver lipid levels, inflammation, oxidative stress levels, and mRNA expression of oxidative metabolism- and inflammation-related genes in mice that had been pretreated with Lactobacillus plantarum versus control mice that had not been pretreated. Our results showed that L. plantarum ZS62 attenuated alcohol-induced weight loss; prevented morphological changes in hepatocytes; reduced markers of liver damage including aspartate aminotransaminase (AST), alanine aminotransaminase (ALT), hyaluronidase (HAase), precollagen III (PC III), and inflammatory cytokines; and enhanced the antioxidative status. L. plantarum ZS62 also significantly downregulated inflammation-related genes and upregulated lipid- and oxidative-metabolism genes. Thus, Lactobacillus plantarum pretreatment appears to confer hepatic protection by reducing inflammation and enhancing antioxidative capacity. The protective effect of L. plantarum ZS62 was even better than that of a commonly used commercial lactic acid bacteria (Lactobacillus delbrueckii subsp. Bulgaricus). The L. plantarum ZS62 might be a potentially beneficial prophylactic treatment for people who frequently drink alcoholic beverages.

\section{Introduction}

Lactobacillus plantarum is one of the lactic acid bacteria (LAB) that can be easily isolated from plant-based foods and naturally fermented foods. It has been widely used in the food industry, as well as in the manufacturing of health products. The strains used in functional foods usually have health-promoting characteristics, such as Lactobacillus plantarum P-8 [1], L. plantarum FLPLo5 [2], and L. plantarum LP3 [3]. These three strains are classified as probiotics which can be beneficial when consumed in adequate amounts (FAO/WHO). More and more strains have been shown to possess benefits in models of cardiovascular disease $[4,5]$, cancer [6], and liver disease $[7,8]$.
The liver is the primary organ of alcohol metabolism that is easily damaged by chronic and excessive alcohol consumption. ALD is a wide spectrum of liver lesions, ranging from steatosis to fibrosis/cirrhosis. ALD is a global healthcare problem that might be one of the oldest diseases of human beings due to alcoholic beverages that existed as early as circa 10,000 B.C [9]. In 2016, alcohol resulted in $5.3 \%$ of all deaths (some 3 million) worldwide. In China, $6 \%$ of males and $1 \%$ of females die from alcohol-related diseases [10]. Moreover, 51.1 males and 27.1 females per 100,000 population, respectively, have liver cirrhosis [11]. Simple alcoholic fatty liver is usually self-limited, and asymptomatic and can be recovered by abstinence. There are $5 \%$ to $15 \%$ patients, however, who still progress to 
fibrosis and cirrhosis after abstinence [12]. The development/progression of ALD is affected by dose, drinking patterns, duration, and so forth, among which the total amount of ingested alcohol is the most important factor [13]. Another important factor, binge drinking pattern, raises the risk of ALD, as well as the all-cause mortality [14]. Enzymes involved in the metabolism of alcohol, including alcohol dehydrogenase and acetaldehyde dehydrogenase, are also associated with the development of ALD.

The pathogenesis of ALD is not fully understood. Much evidence has suggested that probiotics may have the ability to prevent or treat ALD without side effects [15]. For example, Lactobacillus rhamnosus CCFM1107 [16], L. fermentum LA12 [17], and L. plantarum LC27 [18] can attenuate alcohol-induced liver injury. Furthermore, even heat-killed strains and breaking solutions can ameliorate ALD [19, 20]. Numerous studies have reported that probiotics can attenuate alcoholic liver damaged mainly via improving lipid metabolism [21], reducing TNF- $\alpha$ [22], IL-6 [23], and IL-1 $\beta$ [24], and ameliorating oxidative stress [25]. Reducing oxidative stress includes reducing MDA levels, increasing SOD activity [23], and regulating the keep-Nrf2-ARE signaling pathway [20].

In this study, we investigated if Lactobacillus plantarum ZS62 has antioxidative and anti-inflammation effects in a model of alcohol-induced hepatic injury in mice. Regulatory effects on the related gene expression were explored to further elucidate the mechanism of hepatic protection conferred by this bacterial strain. This avenue of investigation may help to develop beneficial probiotic products for consumption by humans.

\section{Materials and Methods}

2.1. Strains. L. plantarum ZS62 was isolated from naturally fermented yogurt in Zhaosu, Xinjiang, China, identified in NCBI based on the Basic Local Alignment Search Tool (BLAST), and preserved in the China General Microbiological Culture Collection Center (CGMCC, No. 18228). A commonly used commercial strain, Lactobacillus delbrueckii subsp. bulgaricus (CGMCC No. 1.16075), was purchased as a comparison. Both strains were stored at $-80^{\circ} \mathrm{C}$ and activated in MRS liquid medium for $24 \mathrm{~h}$ before use; the cultures were centrifuged $(10 \mathrm{~min}, 3000 \mathrm{rpm})$ to collect cells and resuspended in normal saline.

2.2. Mice Models of Alcohol-Induced Subacute Hepatic Damage. Male Kunming mice [26] (forty, 6-week-old), purchased from Experimental Animal Center of Chongqing Medical University (Chongqing, China), were acclimated to the appropriate environment $\left(25 \pm 2{ }^{\circ} \mathrm{C}\right.$, relative humidity $55 \pm 5 \%$, and 12-h day/night cycle), with food and water provided ad libitum. After 7 days' adaptation, mice were randomly grouped $(n=10)$ into the control group (control), model group (model), alcohol and Lactobacillus delbrueckii subsp. bulgaricus fed group (alcohol+LDSB), and alcohol and L. plantarum ZS62 fed group (alcohol+ZS62).

During the experiment, all groups received normal diets, twice daily gavage administration, and daily weightings. At
9:00 am, mice in the control and model group received gavage administration of $0.85 \%(\mathrm{v} / \mathrm{v})$ saline solution, and alcohol+LDSB and alcohol+ZS62 received gavage administration of LDSB and L. plantarum ZS62, respectively; 3 hours later $(12: 00 \mathrm{am})$, mice in the control group also received gavage administration of $0.85 \%(\mathrm{v} / \mathrm{v})$ saline solution, and mice in the model, alcohol+LDSB and alcohol+ZS62, received gavage administration of alcohol (Figure 1). The $56^{\circ}$ liquor ( $56 \%$ alcoholic beverage, v/v) was given at $0.13 \mathrm{~mL} / 10 \mathrm{~g} \mathrm{BW}$ and provided $5.82 \mathrm{~g}_{\text {alcohol }} / \mathrm{kg}_{\mathrm{bw}}$. On the eighth day, all the groups were euthanized (fasted for $12 \mathrm{~h}$ ) before the treatment in Figure 1 (the first gavage administrations were also performed at 9:00 am, and the second gavage administrations were performed and 11:00 am). The viscera (heart, kidney, and liver) were obtained to calculate the organ indexes (viscus weight/body weight). Blood was collected and centrifuged $\left(4^{\circ} \mathrm{C}, 3500 \mathrm{rpm}, 10 \mathrm{~min}\right)$ for serum preparation. The liver was homogenized (10\% homogenate) and centrifuged $\left(4^{\circ} \mathrm{C}\right.$, $4000 \mathrm{rpm}, 10 \mathrm{~min}$ ) to obtain the supernatant.

2.3. Morphological Observation. Liver samples were fixed in formalin $(10 \%, \mathrm{v} / \mathrm{v})$, dehydrated, embedded in paraffin, and sliced. Then, the morphologies of samples were observed after been stained by hematoxylin and eosin (H\&E) method.

2.4. Detections of Serum Indexes. The enzyme activities of AST, ALT, HAase, and PC III and contents of interleukin(IL-) 10 , IL- $1 \beta$, IL-6, and tumor necrosis factor- $\alpha$ (TNF- $\alpha$ ) in serum were measured using purchased kits (Nanjing Jiancheng Bioengineering Institute, Nanjing, Jiangsu, China).

2.5. Measurement of Liver Indexes. The liver levels of total cholesterol (TC), triglyceride (TG), enzyme activities of alcohol dehydrogenase $(\mathrm{ADH})$ and acetaldehyde dehydrogenase $(\mathrm{ALDH})$, and oxidative stress-related indexes of malondialdehyde (MDA), superoxide dismutase (SOD), and glutathione (GSH) were detected by purchased kits (Nanjing Jiancheng Bioengineering Institute, Nanjing, Jiangsu, China).

2.6. Measurement of the mRNA Expression. The hepatic total RNA was extracted by the TRIzolTM Reagent method [27]. Relative mRNA expression of C-Jun N-terminal kinase (JNK), extracellular regulated protein kinases (ERK), cyclooxygenase-1 (COX1), peroxisome proliferator-activated receptor- $\alpha$ (PPAR- $\alpha$ ), nuclear factor erythroid-2 related factor 2 (Nrf2), heme oxygenase-1 (HO-1), SOD1, SOD2, glutathione peroxidase (GSH-Px), catalase (CAT), and nicotinamide adenine dinucleotide phosphate (NADPH) were detected by quantitative real-time reverse transcription-polymerase chain reaction (RT-PCR). Glyceraldehyde-3-phosphate dehydrogenase $(\mathrm{GAPDH})$ also was detected as an internal reference. The purity and concentration of RNA were determined, adjusted to $1 \mu \mathrm{g} / \mu \mathrm{L}$, and used as a template in reverse transcription PCR $(20 \mu \mathrm{L}$ reaction system). The relative gene expression was calculated according to the $2^{-\Delta \Delta \mathrm{Ct}}$ method [27].

2.7. Statistical Analysis. The determinations were performed in triplicate and presented as the mean \pm standard deviation. The data were analyzed (SPSS Inc., Chicago, IL, USA), and 


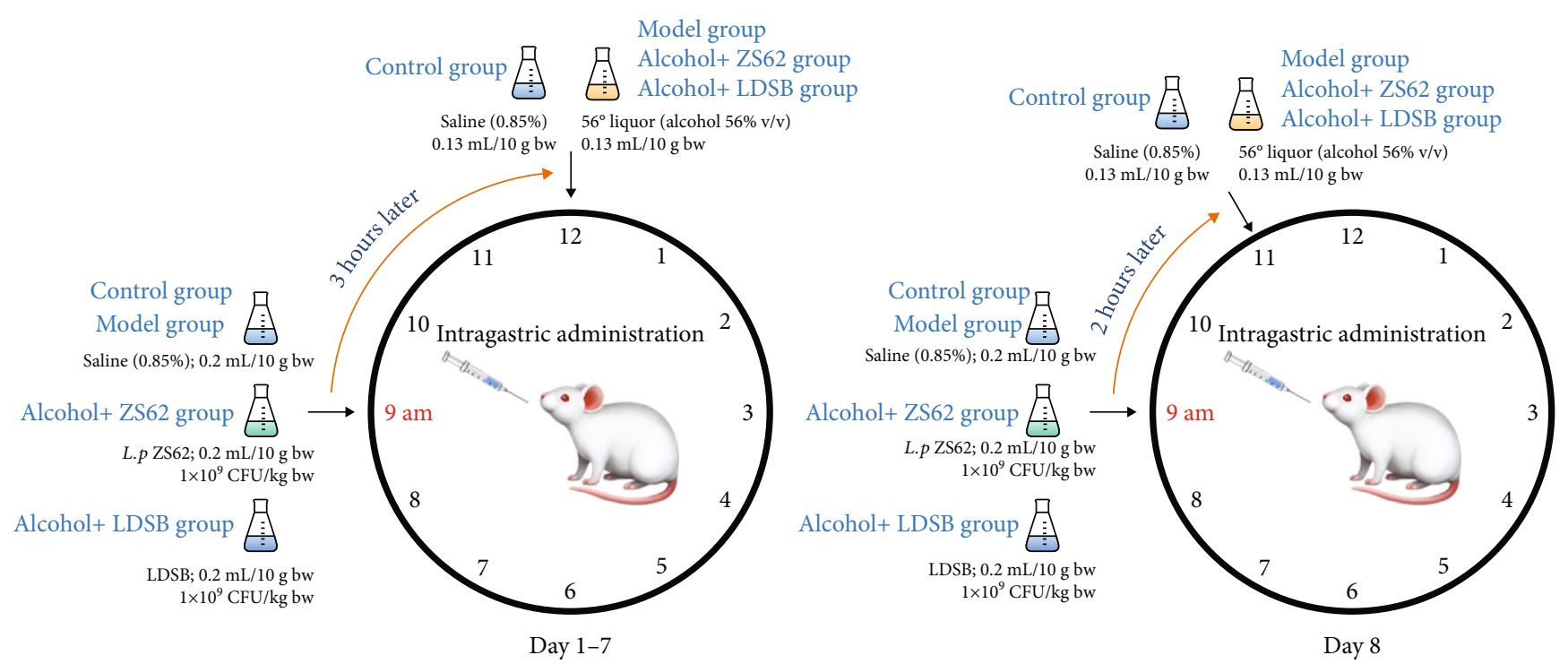

Figure 1: Treatments during the experiment. Control: mice without treatments except twice $0.85 \%$ saline treatment a day; model: mice treated with alcohol $\left(56 \%, \mathrm{v} / \mathrm{v}, 0.13 \mathrm{~mL} / 10 \mathrm{~g}_{\mathrm{bw}}\right)$; alcohol+LDSB: mice treated with of $1.0 \times 10^{9} \mathrm{CFU} / \mathrm{kg}_{\mathrm{bw}}$ Lactobacillus delbruechill subsp. Bulgaricus before alcohol treatment; alcohol+ZS62: mice treated with $1.0 \times 10^{9} \mathrm{CFU} / \mathrm{kg}_{\mathrm{bw}}$ Lactobacillus plantrum ZS62 before alcohol treatment.

statistical significance $(p<0.05)$ was determined by a oneway analysis of variance using the Duncan multirange test.

\section{Results}

3.1. Body Weight Variation. During the experiment, the bodyweight of the control group increased from $31.75 \pm$ $1.29 \mathrm{~g}$ to $35.47 \pm 1.34 \mathrm{~g}$ (Figure $2(\mathrm{a})$ ), while that of the model significantly decreased from $32.13 \pm 1.59 \mathrm{~g}$ to $23.62 \pm 1.09 \mathrm{~g}$ $(p<0.05)$. At the same time, alcohol+LDSB and alcohol+ZS62 also decreased $25.33 \pm 1.09 \mathrm{~g}$ and $30.38 \pm 1.37 \mathrm{~g}$, respectively. However, the magnitude of weight loss was decreased by treatment with L. plantarum ZS62 that significantly lighter than those of model and alcohol+LDSB $(p<0.05)$.

3.2. Organ Indexes of the Mice. The organ indexes are shown in Figure 2(b). The liver proportion to body weight of control $(4.14 \pm 0.43 \%)$ was significantly lower than that of model (4.96 $\pm 0.38 \% ; p<0.05)$. The liver index of alcohol+ZS62 $(4.32 \pm 0.09 \%)$ was significantly lower than that of model $(p<0.05)$, while that of alcohol+LDSB $(4.75 \pm 0.44 \%)$ was at the level of model $(p>0.05)$. The kidney and heart index of each group, however, was the same $(p>0.05)$.

3.3. Liver Pathological Observation. The stained liver tissue slices $(\times 100, \times 200)$ were shown in Figure 3. Hepatic morphology of the control group exhibited normal organized liver structures and lobular architecture, centered nuclei, and uniform cell size, while the structures of mice from the model group were disorganized, with no clearly defined boundaries nor centered nuclei. Moreover, significant hepatic inflammation was observed in the model group. The alcohol+LDSB showed a similar morphology to the model, but the inflammation stratus was alleviated to some degree. At the same time, the morphologies of alcohol+ZS62 were similar to those of control that demonstrated organized structures and clearly defined boundaries.

3.4. Serum Indexes of Each Group. The serum indexes of AST, ALT, HAase, PC III, IL-6, IL- $1 \beta$, and TNF- $\alpha$ in the Model group were markedly elevated relative to the control group and IL-10 which is an anti-inflammatory cytokine was noticeably lower than those of control $(p<0.05$; Figure 4(a)). Both the LAB interventions blocked this alcohol-induced increase in the activities of AST, ALT, and HAase, and concentration of PC III and proinflammatory factor (IL-6, IL-1 $\beta$, and TNF- $\alpha$ ) and significantly increased the concentration of IL-10 $(p<0.05)$. The levels of AST, HAase, and IL-1 $\beta$ of alcohol+ZS62 dropped to the same levels of control $(p>0.05)$. The concentration of IL-10 of alcohol+LDSB was significantly higher than that of alcohol+ZS62 $(p<0.05)$.

3.5. Liver Indexes of Each Group. The hepatic concentration of TC and TG was significantly increased by alcoholic damage $(p<0.05)$. This rise was blocked by treatment with LDSB and L. plantarum ZS62 ( $p<0.05$; Figure 4(b)). Moreover, the level of TG in both LAB intervention groups was reduced to the same level of control $(p>0.05)$. The activities of $\mathrm{ADH}$ and $\mathrm{ALDH}$ were increased by 7-day induction by alcohol $(p<0.05)$; the activities of both enzymes were further enhanced by treatment with LDSB and L. plantarum ZS62 $(p<0.05)$, and the activity of alcohol+ZS62 was noticeably higher than that of alcohol+LDSB $(p<0.05)$.

The activity of SOD and the concentration of GSH was severely lowered by alcohol which was $69.31 \%$ and $36.17 \%$ of control (697 $\pm 54 \mathrm{U} / \mathrm{mg}$ port, $29.15 \pm 4.54 \mu \mathrm{mol} / \mathrm{mg}$ protein) that consequently elevated the level of MDA in 


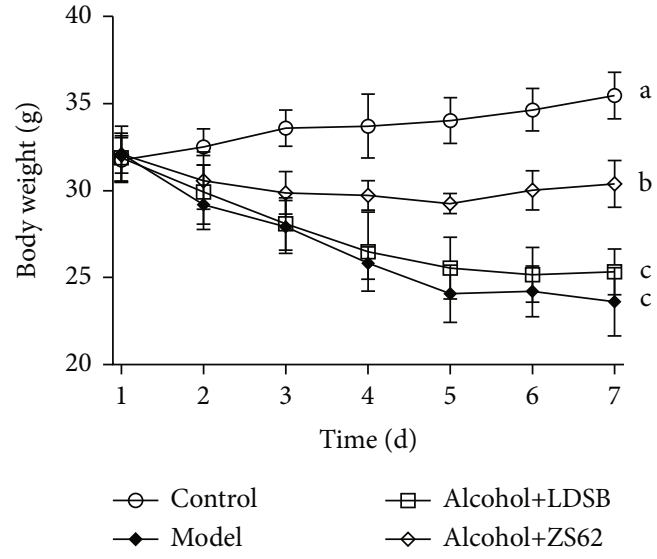

(a)

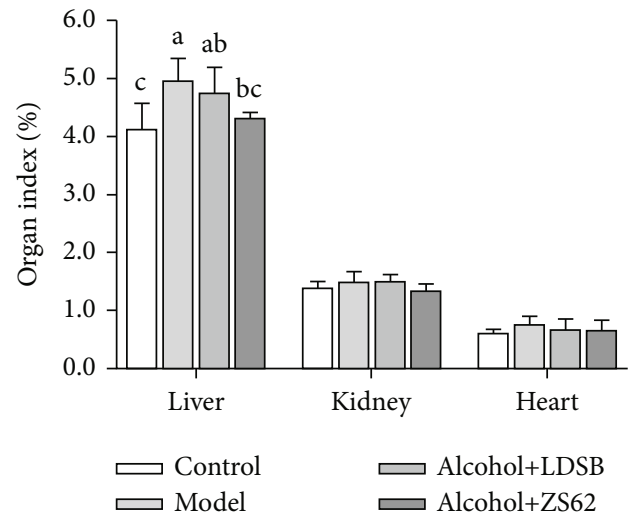

(b)

Figure 2: Changes of body weight (a) and organ indexes (b) of each group. The different alphabets $\left({ }^{\mathrm{a}-\mathrm{c}}\right)$ mean significant differences $(p<0.05)$. Control: mice without treatments except twice $0.85 \%$ saline treatment a day; model: mice treated with alcohol $(56 \%$, v/v, $0.13 \mathrm{~mL} / 10 \mathrm{~g}_{\mathrm{bw}}$ ); alcohol+LDSB: mice treated with of $1.0 \times 10^{9} \mathrm{CFU} / \mathrm{kg}_{\mathrm{bw}}$ Lactobacillus delbruechill subsp. Bulgaricus before alcohol treatment; alcohol+ZS62: mice treated with $1.0 \times 10^{9} \mathrm{CFU} / \mathrm{kg}_{\mathrm{bw}}$ Lactobacillus plantrum ZS62 before alcohol treatment.

the liver $(p<0.05)$. Fortunately, the hepatic activities of SOD in alcohol+LDSB and Aacohol+ZS62 group were increased to $566 \pm 43 \mathrm{U} / \mathrm{mg}$ prot and $603 \pm 56 \mathrm{U} / \mathrm{mg}$ prot, respectively, so was the concentration of GSH that climbed to $16.69 \pm$ $1.80 \mu \mathrm{mol} / \mathrm{mg}$ prot and $21.54 \pm 0.90 \mu \mathrm{mol} / \mathrm{mg}$ prot (LDSB, ZS62, respectively). So, the concentration of MDA in the alcohol+LDSB and alcohol+ZS62 groups dropped significantly $(p<0.05)$.

3.6. Relative mRNA Expression in the Liver. Figure 5 illustrates the effects of alcohol on the expression of mRNA expression of $J N K, E R K$, and $C O X 1$, which were significantly downregulated $(p<0.05)$. The expression of PPAR- $\alpha, N r f 2$, HO-1, SOD1, SOD2, GSH-Px, CAT, and NADPH were markedly upregulated by alcohol $(p<0.05)$ when compared to control. Meanwhile, gavage administration of $L$. plantarum ZS62 strikingly regulated all the genes above $(p<0.05)$ except COX1 and GSH-Px $(p>0.05)$; the LDSB showed a similar but weaker effect on these genes. The expression of $N A D P H$ in both LAB groups was upregulated to the same level of control $(p>0.05)$. There were no significant differences in the expression of COX1 between model and LAB groups; however, the L. plantarum ZS62 also helped to downregulate the expression to the control level $(p>0.05)$. Even the regulation effect of LDSB on CAT was greater than that of $L$. plantarum ZS62 $(p<0.05)$, the overall regulation effect of $L$. plantarum ZS62 was more pronounced than that of LDSB $(p<0.05)$.

\section{Discussion}

Alcoholic beverages include distilled spirits, fermented spirits, mixed spirits, and premixed spirits that contain $0.5 \%$ or higher alcohol content by volume. Alcoholic beverages have been consumed for social entertainment and leisure for thousands of years across the world. However, excess alcohol can lead to multiple behavioral and health problems. Despite the fact that alcohol provides $8.75 \mathrm{kcal} / \mathrm{g}$, the weights of our alcohol-fed mice decreased. The degree of weight loss was closely connected with the drunk durations (time of losing righting reflex) which were $12 \pm 1.4 \mathrm{~h}$, $10 \pm 1.6 \mathrm{~h}$, and $5 \pm 1.6 \mathrm{~h}$ for model, alcohol+LDSB, and alcohol+ZS62, respectively.

The liver is the most important organ for alcohol metabolism. During alcohol metabolism, the production of free radicals results in oxidative stress, as well as inflammation. This damages hepatocyte morphology and function, possibly leading to liver fibrosis [28]. Serum AST and ALT are medical indicators of liver function which significantly increase during liver dysfunction. In the progression of liver fibrosis, HAase and PC III are sensitive indexes that can accurately reflect the degree of liver damage. The increased concentrations of TC and TG in our model of alcohol-induced liver damage also suggested the aggravation of lipid accumulation and disorder of lipid metabolism [29]. The elevated levels of AST, ALT, HAase, and PC III suggest that the liver was injured by the alcohol consumption, and the rising levels of TC and TG show that lipid metabolism was disrupted (Figure 4). The decreased levels of these indexes in mice administered our putative therapeutic suggest that the liver dysfunction has been relieved. Thus, the LDSB and $L$. plantarum ZS62 alleviate the adverse effects of alcohol on liver functions.

The increased inflammatory cytokine levels reflect the level of severity of liver damage. Among these inflammatory cytokines, IL- 6 is initially produced when inflammation occurs. IL-6 is a multifunctional cytokine of the acute phase response [30]. The increased serum IL- 6 indicates that intake of alcohol causes an acute phase response (Figure 4(a)). Studies have described that increased serum IL-6 is associated with alcohol cravings and that IL-6 levels drop during abstinence [31]. TNF- $\alpha$, a neurobiological marker, is related to alcohol consumption and takes part in the regulation of alcohol consumption [32]. However, the phenomenon of eagerness to drink was not observed in this study. Increased levels of IL-1 $\beta$, a proinflammatory cytokine, also could aggravate alcoholic induced inflammation [33], 


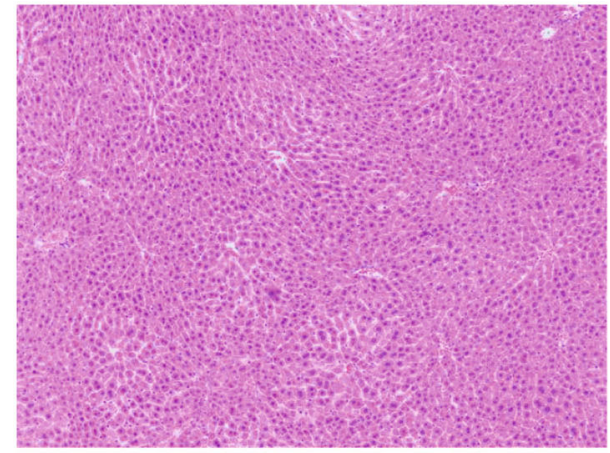

Control $(100 \times)$

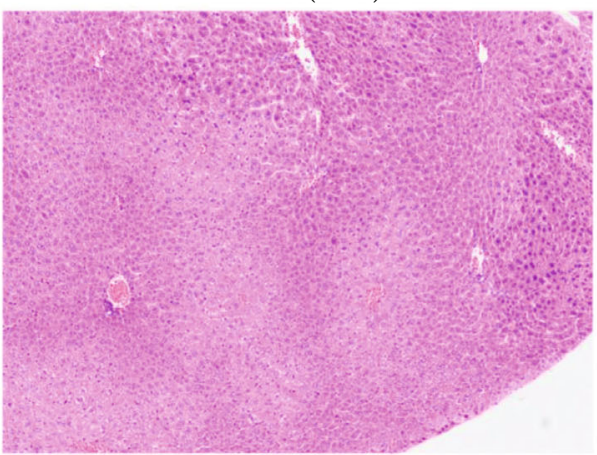

Model (100x)

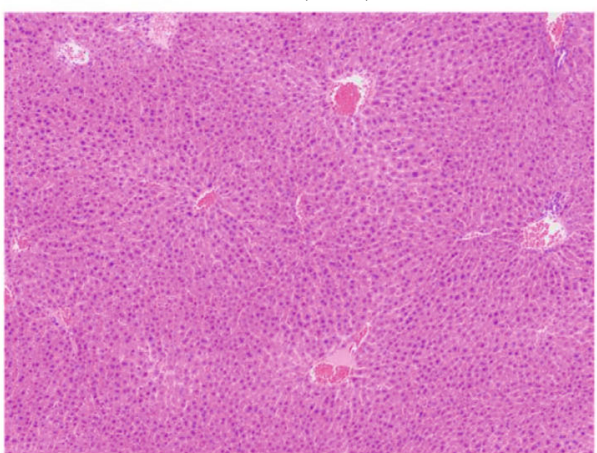

Alcohol+LDSB $(100 \times)$

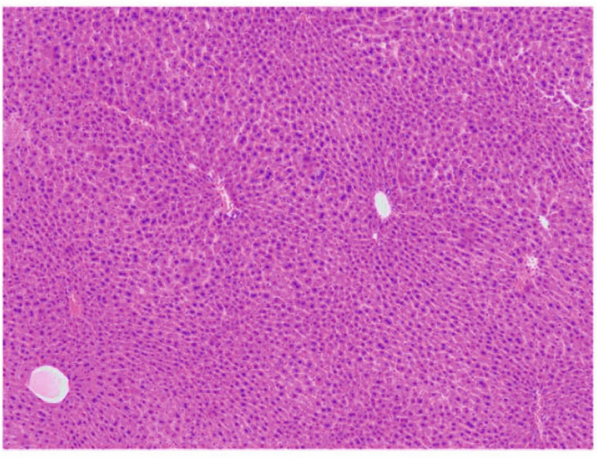

Alcohol+ZS62 (100×)

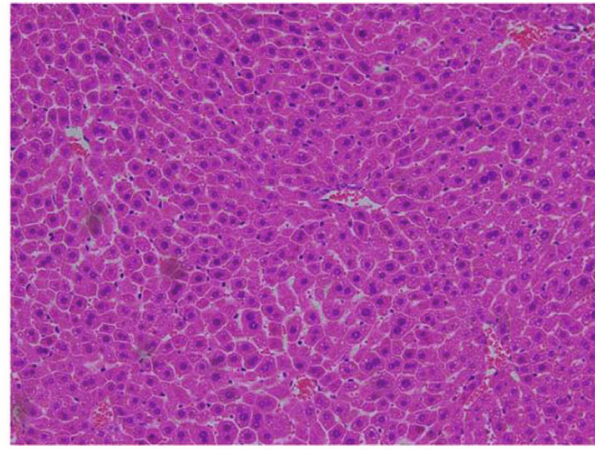

Control $(200 \times)$

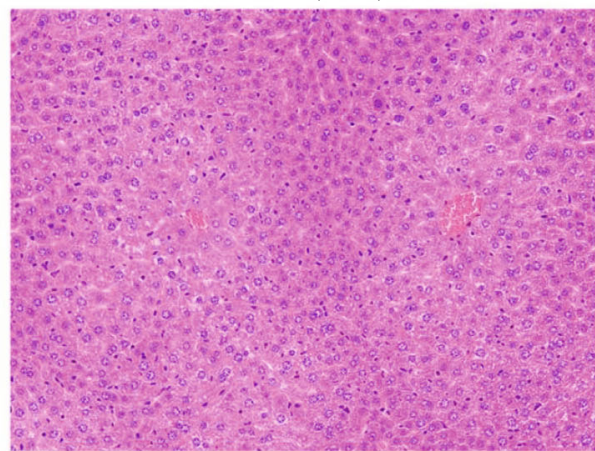

Model (200x)

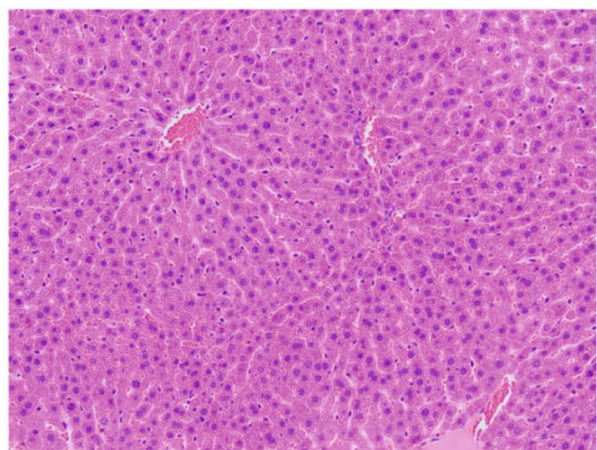

Alcohol+LDSB (200x)

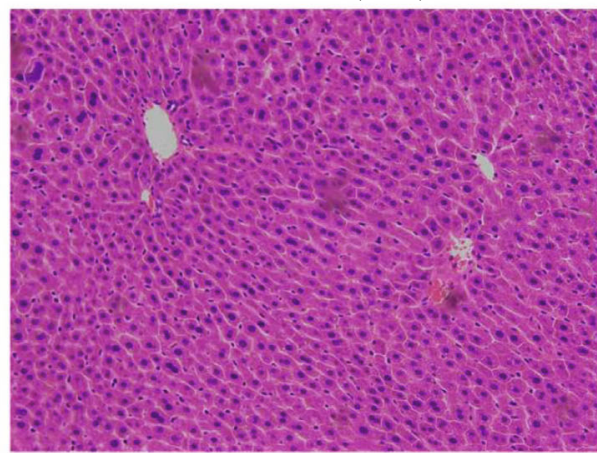

Alcohol+ZS62 (200×)

FIgURE 3: Hematoxylin-eocin (H\&E) staining results of liver in mice. Magnification $\times 100$ and $\times 200$. Control: mice without treatments except twice $0.85 \%$ saline treatment a day; model: mice treated with alcohol (56\%, v/v, $0.13 \mathrm{~mL} / 10 \mathrm{~g}$ bw); alcohol+LDSB: mice treated with of $1.0 \times 10^{9} \mathrm{CFU} / \mathrm{kg}_{\mathrm{bw}}$ Lactobacillus delbruechill subsp. Bulgaricus before alcohol treatment; alcohol+ZS62: mice treated with $1.0 \times 10^{9} \mathrm{CFU} / \mathrm{kg}_{\mathrm{bw}}$ Lactobacillus plantrum ZS62 before alcohol treatment.

while enhancing IL-10, an anti-inflammation cytokine important in immunomodulation after alcohol intake [34]. Our results showed similar results in that the proinflamma- tory cytokines were remarkably increased, and antiinflammation cytokines were decreased by 7-day of induction, and the treatment with LDSB and L. plantarum ZS62 

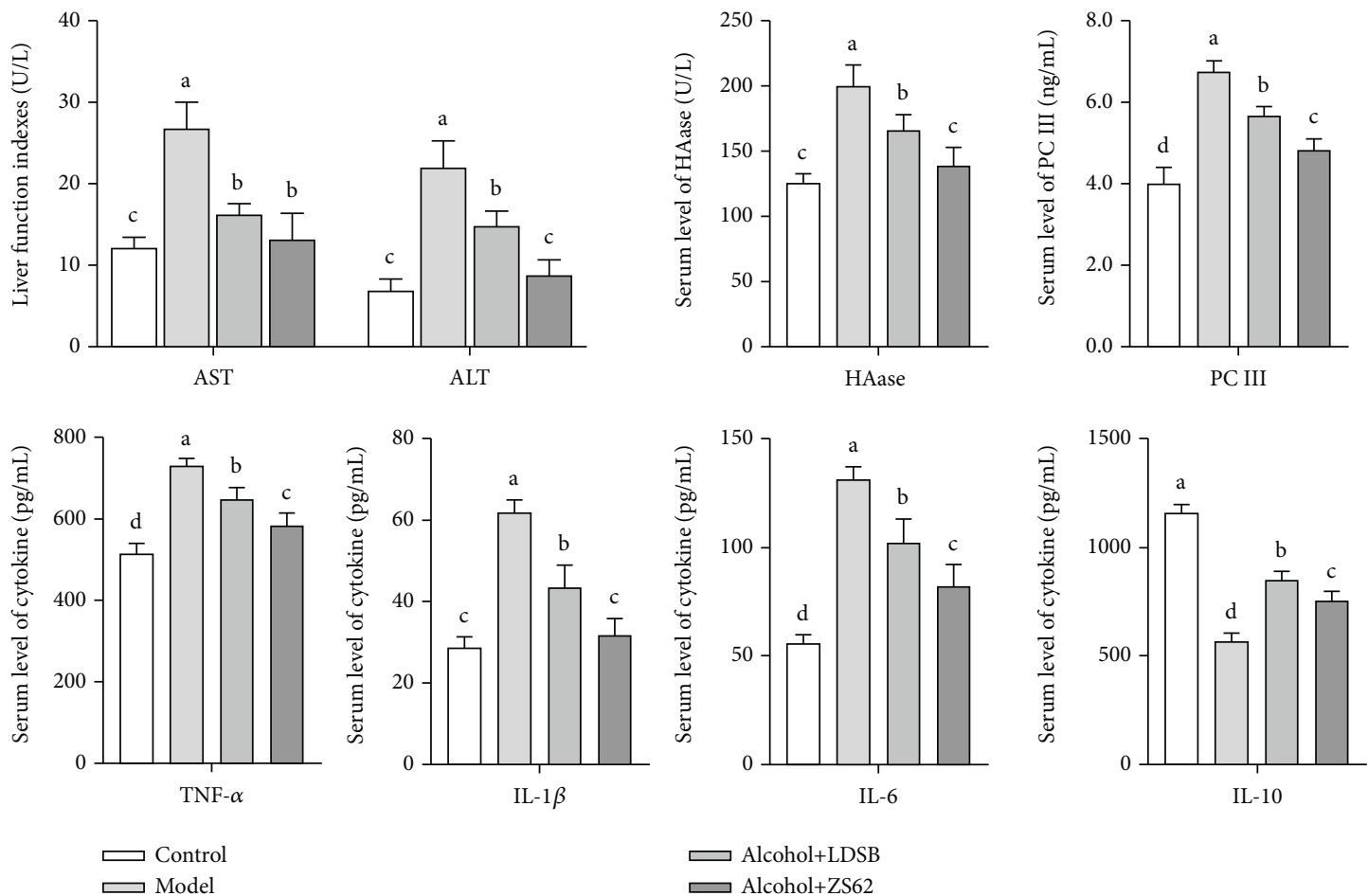

$$
\text { Control }
$$$$
\text { ๑ Alcohol+ZS62 }
$$

(a)
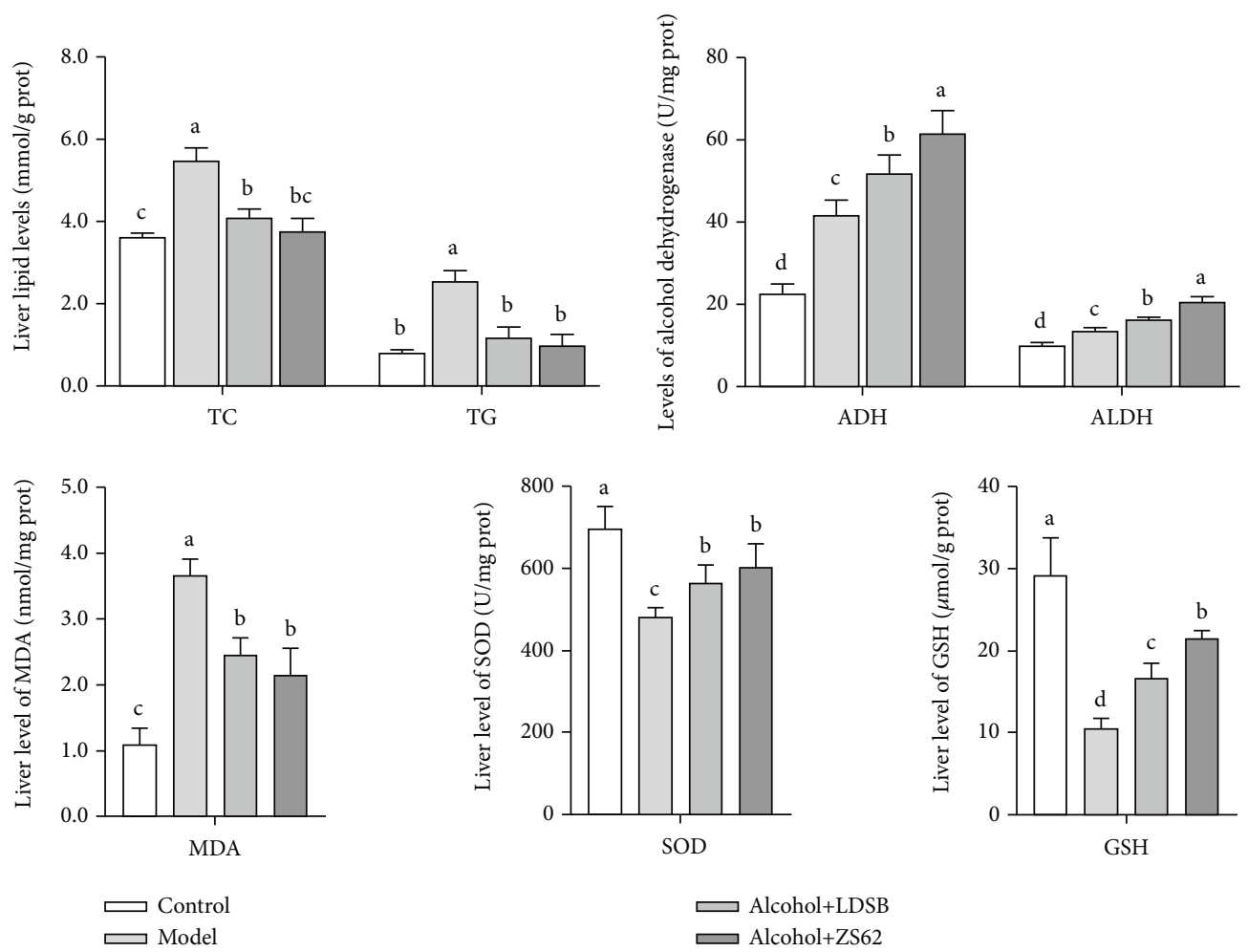

(b)

FIgure 4: Serum and liver indexes of mice in each group: (a) Serum levels of ALT, AST, HAase, PC III, TNF- $\alpha$, IL-6, IL-1 $\beta$, and IL-10. (b) Liver levels of TC, TG, ADH, ALDH, MDA, SOD, and GSH. The different alphabets $\left({ }^{\mathrm{a}-\mathrm{d}}\right)$ mean significant differences $(p<0.05)$. Control: mice without treatments except twice $0.85 \%$ saline treatment a day; model: mice treated with alcohol $(56 \%, \mathrm{v} / \mathrm{v}, 0.13 \mathrm{~mL} / 10 \mathrm{~g}$ bw); alcohol+LDSB: mice treated with of $1.0 \times 10^{9} \mathrm{CFU} / \mathrm{kg}_{\mathrm{bw}}$ Lactobacillus delbruechill subsp. Bulgaricus before alcohol treatment; alcohol+ZS62: mice treated with $1.0 \times 10^{9} \mathrm{CFU} / \mathrm{kg}_{\mathrm{bw}}$ Lactobacillus plantrum ZS62 before alcohol treatment. 

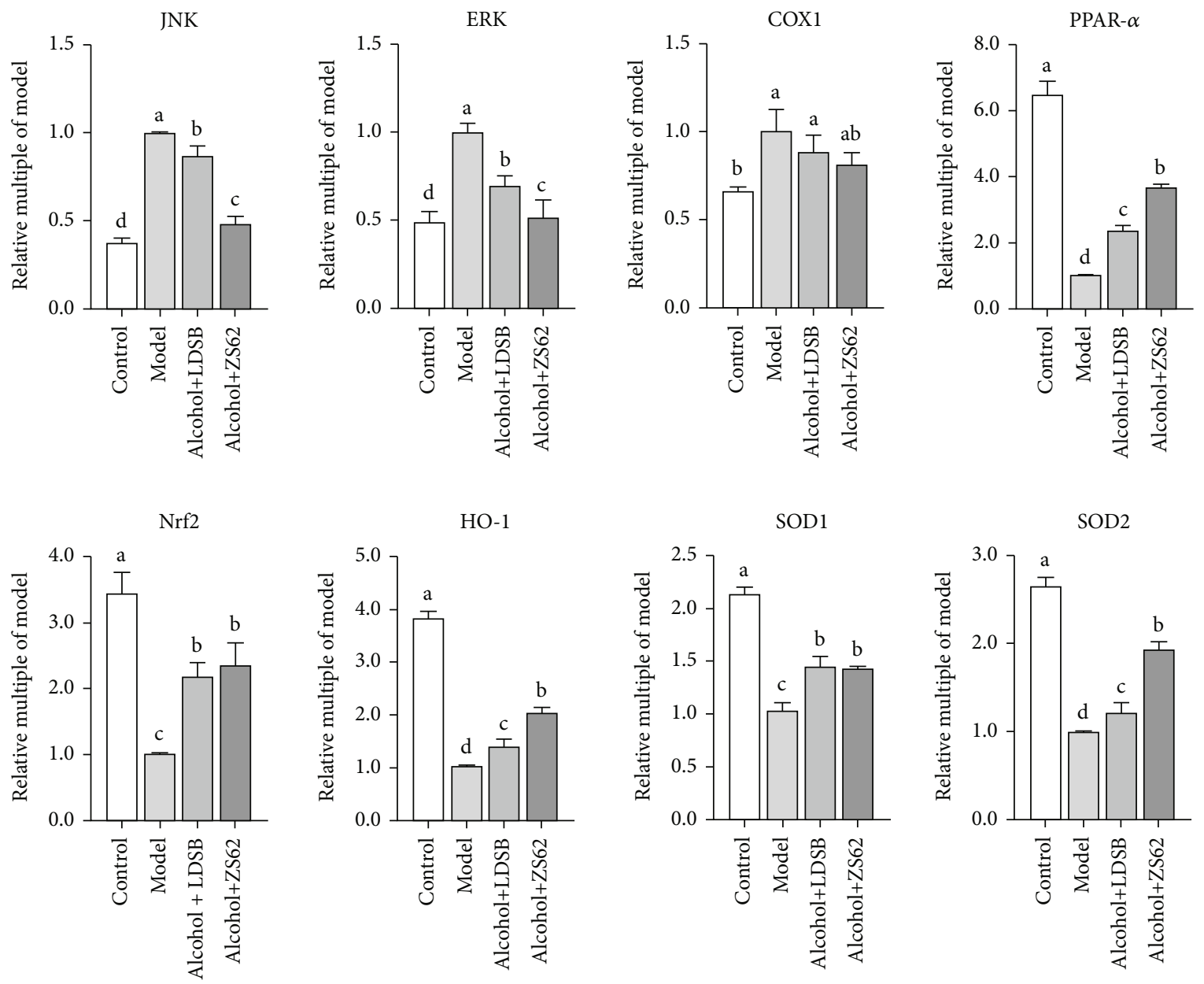

GSH-PX
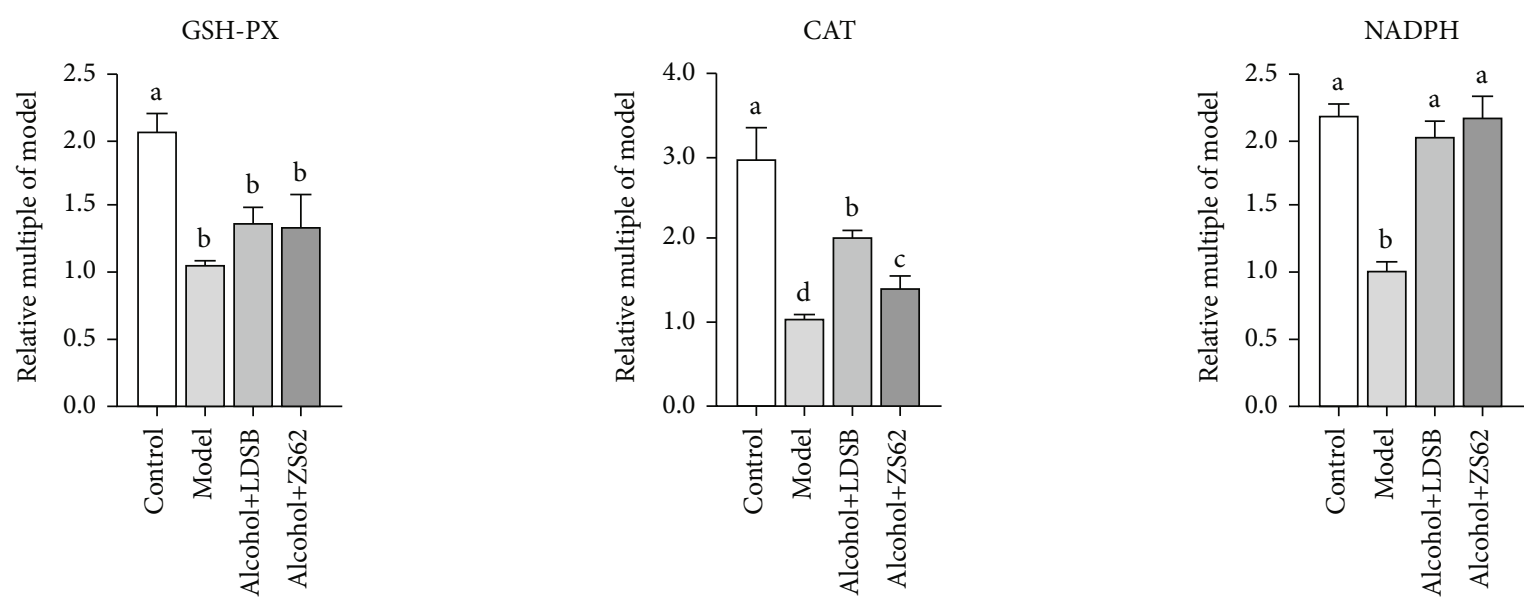

FIGURE 5: Hepatic mRNA expressions of mice; c-Jun N-terminal kinase (JNK), extracellular regulated protein kinases (ERK), cyclooxygenase-1 (COX1), peroxisome proliferator-activated receptor- $\alpha$ (PPAR- $\alpha$ ), nuclear factor erythroid-2 related factor 2 (Nrf2), hemeoxygenase-1 (HO-1), superoxide dismutase 1 (SOD1), superoxide dismutase 2 (SOD2), GSH-Px (glutathione peroxidase), catalase $(\mathrm{CAT})$, and nicotinamide adenine dinucleotide phosphate (NADPH) mRNA. The different alphabets $\left({ }^{\mathrm{a}-\mathrm{d}}\right)$ mean significant differences $(p<0.05)$. Control: mice without treatments except twice $0.85 \%$ saline treatment a day; model: mice treated with alcohol $(56 \%$, v/v, $\left.0.13 \mathrm{~mL} / 10 \mathrm{~g}_{\mathrm{bw}}\right)$; alcohol+LDSB: mice treated with of $1.0 \times 10^{9} \mathrm{CFU} / \mathrm{kg}_{\mathrm{bw}}$ Lactobacillus delbruechill subsp. Bulgaricus before alcohol treatment; alcohol+ZS62: mice treated with $1.0 \times 10^{9} \mathrm{CFU} / \mathrm{kg}_{\mathrm{bw}}$ Lactobacillus plantrum ZS62 before alcohol treatment.

effectively reduced the production of proinflammatory agents and increased the anti-inflammation factor levels (Figure 4(a)). Even the concentration of IL-10 in alco-
hol+LDSB was significantly higher than that of alcohol+ZS62, the measured proinflammatory levels of alcohol+ZS62 were remarkably lower than those of alcohol+LDSB. And combined 
with Figure 3, L. plantarum ZS62 showed an excellent effect on alleviating inflammation status.

$\mathrm{ADH}$ and $\mathrm{ALDH}$ are important enzymes that participate in alcohol metabolism that converts alcohol to acetaldehyde by $\mathrm{ADH}$ and the following are oxidized to carboxylic acids by ALDH. Figure 4(b) demonstrates that alcohol-gavage increased the activates of both enzymes (model), and the $L$. plantarum ZS62 showed a further beneficial effect that helped to accelerate alcohol metabolism that can lessen the damage of acetaldehyde on the liver. These results might explain the attenuated inflammatory status of alcohol+ZS62.

Reactive oxygen species (ROS) are well known to cause oxidative stress when overproduced; the excess acetaldehyde also is converted to superoxide, consequently aggravating oxidative stress [35]. Scavengers of ROS and antioxidants, such as SOD and GSH, are important in reducing oxidative stress on the body. The lipid peroxidation end product, MDA, is also used as a marker of oxidative stress. Figure 4(b) shows that the levels of SOD and GSH in this model were sharply decreased, and the level of MDA was increased, which indicated that the body might be easily attacked by ROS and increased oxidative stress. Abnormal cellular morphology in Figure 3 was consistent with this assumption. At the same time, gavage administration of $L$. plantarum ZS62 significantly enhanced ROS scavenging capacity. The reduced MDA level was also associated with lower concentrations of hepatic TC and TG.

JNK is a subclass of the mitogen-activated protein kinase (MAPK) signaling pathway easily activated by ROS, TNF, and IL. This pathway can promote the occurrence and development of inflammation and the progression of liver fibrosis $[36,37]$. ERK is another important member of the MAPK family activated in the metabolic syndrome that transfers massages into the nucleus and takes part in the processes of physiology and pathology [38]. Both JNK and ERK associate with collagen production and degradation and also could raise IL-6 levels $[39,40]$. COX1, an isozyme of COXs, responds to inflammation and has become a target of inflammation treatment [41]. The upregulated expressions of JNK and ERK (Model) were significantly downregulated by the treatment of L. plantarum ZS62, and the expression of COX1 was somewhat downregulated (Figure 5). Combined with the serum levels of IL- $1 \beta$, IL- 6 , TNF- $\alpha$, and IL-10 in Figure 4, L. plantarum ZS62 showed the ability to be anti-inflammatory.

PPAR- $\alpha$ is a ligand-induced nuclear receptor highly expressed in the liver, closely associated with biooxidation and fatty acid $\beta$-oxidation, and currently is used to treat dyslipidemia for it exerts an anti-inflammatory effect [42]. The relatively higher levels of TC and TG (Figure 4(b)) are associated with the lower expression of PPAR- $\alpha$ which reduced the speed of lipid metabolism and increased lipid accumulation. Both LAB strains upregulated the expression of PPAR$\alpha$ and dropped the concentration of liver TC and TG.

Nrf2 is one of the antioxidant markers that can prevent hepatocytes from necroptosis and a transcription factor for the expression regulation of $\mathrm{HO}-1$ which is another antioxidant marker $[43,44]$. Activated Nrf2 is regarded as response protection against alcohol-induced liver damage. Alcohol downregulated the expression of both Nrf2 and HO- 1 which indicated that the antioxidative capacity of the model was reduced; the intervene of $L$. plantarum ZS62 effectively upregulated the expression of Nrf2 indicating that this strain responds to alcoholic oxidative stress.

SOD is the first detoxification enzyme of superoxide radical neutralization, among which SOD2 exists in the mitochondrial matrix and neutralizes superoxide radicals. SOD1, another species of SOD, converts harmful $\mathrm{H}_{2} \mathrm{O}_{2}$ produced by the dismutation reaction of SOD2 into $\mathrm{O}_{2}$ and $\mathrm{H}_{2}$ [45]. $\mathrm{H}_{2} \mathrm{O}_{2}$ also can be decomposed by CAT and GSH-Px into $\mathrm{O}_{2}$ and $\mathrm{H}_{2} \mathrm{O}$ [46]. Moreover, CAT reduces fat accumulation by degrading hepatic fatty acids without oxidative damage [47]. NADPH is a hydrogen carrier playing the role of reducing agent in biosynthesis and the coenzyme of GSH reductase by which the intracellular content can be kept [48].

The downregulated expression of SOD1, SOD2, GSH-Px, and $C A T$ reduced the activities of antioxidant enzymes and consequently weakens the antioxidant capacity, and the downregulated expression of $N A D P H$ reduced the delivery of hydrogen and the content of GSH that also contributes to alcoholic oxidative stress. At the same time, both L. plantarum ZS62 and LDSB upregulated these genes that significantly enhanced the antioxidative ability of alcohol-fed mice, and the overall regulation of L. plantarum ZS62 on these genes was greater than that of LDSB. All in all, L. plantarum ZS62's hepatoprotective effect against alcoholic subacute liver damage might be attributable to its antioxidant and anti-inflammatory activities.

\section{Conclusions}

This study suggested that a dose of L. plantarum ZS62 at $1.0 \times 10^{9} \mathrm{CFU} / \mathrm{kg}_{\mathrm{BW}}$ could protect the liver from alcoholic damage by inhibiting alcohol-induced weight loss, alleviating inflammatory status and downregulating the expression of related genes, improving liver function, and upregulating the expression of antioxidant-related genes that enhance the antioxidant status. L. plantarum ZS62 probably resists alcohol-induced subacute liver damage via stimulating antioxidative and anti-inflammation pathways. This study suggests that L. plantarum ZS62 might be a potentially useful probiotic strain and that further studies on hepatic protection are warranted.

\section{Data Availability}

The datasets generated for this study are available upon request to the corresponding author.

\section{Ethical Approval}

The protocol for these experiments was approved by the Ethics Committee of Chongqing Collaborative Innovation Center for Functional Food (202006003B), Chongqing, China, and the experimental process was followed the 2010/63/EU directive. 


\section{Conflicts of Interest}

All authors declare that they have no competing interests.

\section{Authors' Contributions}

Yi Gan and Xiufeng Chen are mainly responsible for the content of the experiment and manuscript writing. Ruokun $\mathrm{Yi}$ is mainly involved in data analysis research. Xin Zhao oversaw the research and reviewed the final manuscript. Yi Gan and Xiufeng Chen contributed equally to this work.

\section{Acknowledgments}

This research was funded by the Chongqing University Innovation Research Group Project (CXQTP20033), Science and Technology Projects of Chongqing Education Commission (KJZD-K202001602), and General Program of Natural Science Foundation of Chongqing (cstc2021jcyj-msxmX0070), China.

\section{References}

[1] L. Y. Kwok, Z. Guo, J. Zhang et al., "The impact of oral consumption of lactobacillus plantarum P-8 on faecal bacteria revealed by pyrosequencing," Beneficial Microbes, vol. 6, no. 4, pp. 405-413, 2015.

[2] X. Yu, Y. Li, Q. Wu, N. P. Shah, H. Wei, and F. Xu, "Genomic analysis for antioxidant property of lactobacillus plantarum FLPL05 from Chinese longevity people," Probiotics and Antimicrobial Proteins, vol. 12, no. 4, pp. 1451-1458, 2020.

[3] Z. Ding, A. Hani, W. Li, L.'. Gao, W. Ke, and X. Guo, "Influence of a cholesterol-lowering strainLactobacillus plantarumLP3 isolated from traditional fermented yak milk on gut bacterial microbiota and metabolome of rats fed with a highfat diet," Food \& Function, vol. 11, no. 9, pp. 8342-8353, 2020.

[4] A. Dehkohneh, P. Jafari, and H. Fahimi, "Effects of probiotic Lactobacillus paracasei TD3 on moderation of cholesterol biosynthesis pathway in rats," Iranian Journal of Basic Medical Sciences, vol. 22, no. 9, pp. 1004-1009, 2019.

[5] V. L. O'Morain and D. P. Ramji, "The potential of probiotics in the prevention and treatment of atherosclerosis," Molecular Nutrition \& Food Research, vol. 64, no. 4, article 1900797, 2019.

[6] T. Legesse Bedada, T. K. Feto, K. S. Awoke, A. D. Garedew, F. T. Yifat, and D. J. Birri, "Probiotics for cancer alternative prevention and treatment," Biomedicine \& Pharmacotherapy, vol. 129, no. 20, article 110409, 2020.

[7] M. Hong, D. H. Han, J. Hong, D. J. Kim, and K. T. Suk, "Are probiotics effective in targeting alcoholic liver diseases?," Probiotics and Antimicrobial Proteins, vol. 11, no. 2, pp. 335$347,2019$.

[8] F. M. F. Elshaghabee, N. Rokana, H. Panwar, K. J. Heller, and J. Schrezenmeir, "Probiotics for dietary management of nonalcoholic fatty liver disease," Environmental Chemistry Letters, vol. 17, no. 4, pp. 1553-1563, 2019.

[9] C. H. Patrick, Alcohol, Culture, and Society, Duke University Press, Durham, NC, 1952.

[10] WHO, Global Status Report on Alcohol and Health, WHO, Geneva, Switzerland, 2018.
[11] "WHO report Alcohol consumption in India doubled in 11 years," 2021, https://www.livemint.com/Industry/ 0PBqBWHOYz8msKWSD6a84H/Alcohol-consumption-inIndia-doubled-in-11-years-WHO-report.html.

[12] R. S. O'Shea, S. Dasarathy, A. J. McCullough, Practice Guideline Committee of the American Association for the Study of Liver Diseases, and Practice Parameters Committee of the American College of Gastroenterology, "Alcoholic liver disease," Hepatology, vol. 51, no. 1, pp. 307-328, 2010.

[13] V. T. Savolainen, K. Liesto, A. Männikkö, A. Penttilä, and P. J. Karhunen, "Alcohol consumption and alcoholic liver disease: evidence of a threshold level of effects of ethanol," Alcoholism: Clinical and Experimental Research, vol. 17, no. 5, pp. 11121117, 1993.

[14] E. Barrio, S. Tomé, I. Rodríguez et al., "Liver disease in heavy drinkers with and without alcohol withdrawal syndrome," Alcoholism: Clinical and Experimental Research, vol. 28, no. 1, pp. 131-136, 2004.

[15] T. Vaikunthanathan, N. Safinia, G. Lombardi, and R. I. Lechler, "Microbiota, immunity and the liver," Immunology Letters, vol. 171, no. 171, pp. 36-49, 2016.

[16] F. Tian, F. Chi, G. Wang et al., "Lactobacillus rhamnosus CCFM1107 treatment ameliorates alcohol-induced liver injury in a mouse model of chronic alcohol feeding," Journal of Microbiology, vol. 53, no. 12, pp. 856-863, 2015.

[17] B. K. Kim, I. O. Lee, P. L. Tan, J. Y. Eor, J. K. Hwang, and S. H. Kim, "Protective effect of Lactobacillus fermentum LA12 in an alcohol-induced rat model of alcoholic steatohepatitis," Korean Journal for Food Science of Animal Resources, vol. 37, no. 6, pp. 931-939, 2017.

[18] W. G. Kim, H. Kim, E. Kwon, M. J. Han, and D. H. Kim, "Lactobacillus plantarum LC27 and Bifidobacterium longum LC67 mitigate alcoholic steatosis in mice by inhibiting LPS-mediated NF- $\kappa \mathrm{B}$ activation through restoration of the disturbed gut microbiota," Food \& Function, vol. 9, no. 8, pp. 4255-4265, 2018.

[19] S. Segawa, Y. Wakita, H. Hirata, and J. Watari, "Oral administration of heat-killed Lactobacillus brevis SBC8803 ameliorates alcoholic liver disease in ethanol-containing diet-fed C57BL/ $6 \mathrm{~N}$ mice," International Journal of Food Microbiology, vol. 128, no. 2, pp. 371-377, 2008.

[20] Y. Liu, X. Liu, Y. Wang et al., "Protective effect of Lactobacillus plantarum on alcoholic liver injury and regulating of keapNrf2-ARE signaling pathway in zebrafish larvae," PLoS One, vol. 14, no. 9, article e0222339, 2019.

[21] X. Li, Y. Liu, X. Guo, Y. Ma, H. Zhang, and H. Liang, "Effect of Lactobacillus casei on lipid metabolism and intestinal microflora in patients with alcoholic liver injury," European Journal of Clinical Nutrition, vol. 75, no. 8, pp. 1227-1236, 2021.

[22] Y. Wang, Y. Liu, I. Kirpich et al., "Lactobacillus rhamnosus GG reduces hepatic TNF $\alpha$ production and inflammation in chronic alcohol-induced liver injury," The Journal of Nutritional Biochemistry, vol. 24, no. 9, pp. 1609-1615, 2013.

[23] L. Zhao, Y. Jiang, Y. Ni et al., "Protective effects of Lactobacillus plantarum C88 on chronic ethanol-induced liver injury in mice," Journal of Functional Foods, vol. 35, pp. 97-104, 2017.

[24] T. Fang, J. Guo, M. Lin, M. S. Lee, Y. L. Chen, and W. H. Lin, "Protective effects of Lactobacillus plantarum against chronic alcohol-induced liver injury in the murine model," Applied Microbiology and Biotechnology, vol. 103, no. 20, pp. 85978608, 2019. 
[25] C. B. Forsyth, A. Farhadi, S. M. Jakate, Y. Tang, M. Shaikh, and A. Keshavarzian, "Lactobacillus GG treatment ameliorates alcohol-induced intestinal oxidative stress, gut leakiness, and liver injury in a rat model of alcoholic steatohepatitis," Alcohol, vol. 43, no. 2, pp. 163-172, 2009.

[26] F. Wang, Y. J. Zhang, Y. Zhou et al., "Effects of beverages on alcohol metabolism: potential health benefits and harmful impacts," International Journal of Molecular Sciences, vol. 17, no. 3, pp. 354-365, 2016.

[27] Y. Gan, J. Liang, W. Diao et al., "Lactobacillus plantarumKSFY06 and geniposide counteract montmorilloniteinduced constipation in Kunming mice," Food Science and Nutrition, vol. 8, no. 9, pp. 5128-5137, 2020.

[28] Y. Yu, X. Sun, J. Gu et al., "Deficiency of DJ-1 ameliorates liver fibrosis through inhibition of hepatic ROS production and inflammation," International Journal of Biological Sciences, vol. 12, no. 10, pp. 1225-1235, 2016.

[29] Y. Zhu, J. Y. Zhang, Y. L. Wei et al., "The polyphenol-rich extract from chokeberry (Aronia melanocarpa L.) modulates gut microbiota and improves lipid metabolism in dietinduced obese rats," Nutrition \& Metabolism, vol. 17, no. 1, pp. 54-69, 2020.

[30] D. Schmidt-Arras and S. Rose-John, "IL-6 pathway in the liver: from physiopathology to therapy," Journal of Hepatology, vol. 64, no. 6, pp. 1403-1415, 2016.

[31] A. Zgierska, D. Rabago, M. Zuelsdorff, C. Coe, M. Miller, and M. Fleming, "Mindfulness meditation for alcohol relapse prevention: a feasibility pilot study," Journal of Addiction Medicine, vol. 2, no. 3, pp. 165-173, 2008.

[32] A. Heberlein, M. Käser, R. Lichtinghagen et al., "TNF- $\alpha$ and IL-6 serum levels: neurobiological markers of alcohol consumption in alcohol-dependent patients?," Alcohol, vol. 48, no. 7, pp. 671-676, 2014.

[33] C. Jiang, Y. Zhang, X. Tang et al., "IL-6 and IL- $1 \beta$ upregulation and tau protein phosphorylation in response to chronic alcohol exposure in the mouse hippocampus," Neuroreport, vol. 32, no. 10, pp. 851-857, 2021.

[34] O. Norkina, A. Dolganiuc, T. Shapiro, K. Kodys, P. Mandrekar, and G. Szabo, "Acute alcohol activates STAT3, AP-1, and Sp-1 transcription factors via the family of Src kinases to promote IL-10 production in human monocytes," Journal of Leukocyte Biology, vol. 82, no. 3, pp. 752-762, 2007.

[35] M. Wang, X. Zhang, F. Liu et al., "Saponins isolated from the leaves of _Panax notoginseng_ protect against alcoholic liver injury via inhibiting ethanol-induced oxidative stress and gut-derived endotoxin-mediated inflammation," Journal of Functional Foods, vol. 19, pp. 214-224, 2015.

[36] E. Hernández-Aquino, M. A. Quezada-Ramírez, A. Silva-Olivares et al., "Naringenin attenuates the progression of liver fibrosis via inactivation of hepatic stellate cells and profibrogenic pathways," European Journal of Pharmacology, vol. 865, article 172730, 2019.

[37] E. D. Hernandez, A. J. Lee, J. Y. Kim et al., "A macrophage NBR1-MEKK3 complex triggers JNK-mediated adipose tissue inflammation in obesity," Cell Metabolism, vol. 20, no. 3, pp. 499-511, 2014.

[38] E. Seki, D. A. Brenner, and M. Karin, "A liver full of JNK: signaling in regulation of cell function and disease pathogenesis, and clinical approaches," Gastroenterology, vol. 143, no. 2, pp. 307-320, 2012.
[39] X. Xu, R. Jiang, M. Chen et al., "Puerarin decreases collagen secretion in angII-induced atrial fibroblasts through inhibiting autophagy via the JNK-Akt-mTOR signaling pathway," Journal of Cardiovascular Pharmacology, vol. 73, no. 6, pp. 373$382,2019$.

[40] Z. Malekpour-Dehkordi, S. Teimourian, M. Nourbakhsh, Y. Naghiaee, R. Sharifi, and J. Mohiti-Ardakani, "Metformin reduces fibrosis factors in insulin resistant and hypertrophied adipocyte via integrin/ERK, collagen VI, apoptosis, and necrosis reduction," Life Sciences, vol. 233, no. 15, article 116682, 2019.

[41] S. H. Choi, R. Langenbach, and F. Bosetti, "Genetic deletion or pharmacological inhibition of cyclooxygenase-1 attenuate lipopolysaccharide- induced inflammatory response and brain injury," FASEB Journal, vol. 22, no. 5, pp. 1491-1501, 2008.

[42] F. Zandbergen and J. Plutzky, "PPAR $\alpha$ in atherosclerosis and inflammation," Biochimica et Biophysica Acta (BBA)-Molecular and Cell Biology of Lipids, vol. 1771, no. 8, pp. 972-982, 2007.

[43] C. Lu, W. Xu, F. Zhang, J. Shao, and S. Zheng, "Nrf2 knockdown disrupts the protective effect of curcumin on alcoholinduced hepatocyte necroptosis," Molecular Pharmaceutics, vol. 13, no. 12, pp. 4043-4053, 2016.

[44] Z. Rahma, D. K. Dwivedi, and G. B. Jena, "Ethanol-induced gastric ulcer in rats and intervention of tert-butylhydroquinone: involvement of Nrf2/HO-1 signalling pathway," Human \& Experimental Toxicology, vol. 39, no. 4, article 096032711989555, 2020.

[45] C. Cruciani-Guglielmacci and C. Magnan, "Brain lipoprotein lipase as a regulator of energy balance," Biochimie, vol. 143, pp. 51-55, 2017.

[46] J. R. Pérez-Estrada, D. Hernández-García, F. Leyva-Castro et al., "Reduced lifespan of mice lacking catalase correlates with altered lipid metabolism without oxidative damage or premature aging," Free Radical Biology Medicine, vol. 135, pp. 102-115, 2019.

[47] H. Lee, J. Lee, T. Alves et al., "Mitochondrial-targeted catalase protects against high-fat diet-induced muscle insulin resistance by decreasing intramuscular lipid accumulation," Diabetes, vol. 66, no. 8, pp. 2072-2081, 2017.

[48] X. Wen, K. Iwata, K. Ikuta et al., "NOX1/NADPH oxidase regulates the expression of multidrug resistance-associated protein 1 and maintains intracellular glutathione levels," FEBS Journal, vol. 286, no. 4, pp. 678-687, 2019. 